\title{
Effect of Nano-Hydroxyapatite Graft on Socket Healing after Teeth Extraction
}

\author{
W.Ghanem ${ }^{1}$, I.Hussien ${ }^{2}$, O.Kilany ${ }^{2}$, I.ELlaboudy ${ }^{3}$ \\ ${ }^{1}$ Department of oral and maxillofacial surgery Faculty of Dentistry, Suez Canal University \\ ${ }^{2}$ Department of Surgery, Anesthesiology and Radiology, Faculty of Veterinary Medicine, \\ Suez Canal University \\ ${ }^{2}$ Department of Clinical Pathology Faculty of Veterinary Medicine Suez Canal University
}

\begin{abstract}
:
Purpose: The aim of the present study was to evaluate hard tissue healing following application of bone substitutes (Nano bone) into fresh extraction sockets in dogs.
\end{abstract}

Methods: The study was conducted on twenty eight mature (ranged from 1-2 years old) male Mongrel dogs, weighing about 15-20 kg each.The following groups were tested:

Group I (control group): consisted of four animals with extracted lower $2^{\text {nd }}$ premolar.

Group II: consisted of twelve animals; the animals in this group were treated with nano hydroxyapatite (NanoBone ${ }^{\circledR)}$ to seal the socket after simple atraumatic extraction of lower $2^{\text {nd }}$ premolar.

Group III: consisted of twelve animals; the animals in this group were treated with nano hydroxyapatite $\left(\right.$ NanoBone $\left.{ }^{\circledR}\right)$ with platelet rich fibrin $(P R F)$ to seal the socket after simple atraumatic extraction of lower $2^{\text {nd }}$ premolar.

One animal from group I and three animals from group II and III were sacrificed with an overdose of anesthesia at 15, 30,45and 90 day.

Results: Healing was more rapid in the group III, II than group I. Histological analysis revealed woven bone filled the socket earliest in groupIII ,II then group I in intervals 30and 45 days. lamellar bone also Seen early in group III, II than group I at the end of 90 day.

Conclusion: Nanobone graft alone and Nanobone graft with the growth factor Platelet Rich Fibrin (PRF) accelerate the formation of bone filling the extracted bony socket and preserved three dimensions of extracted bony socket after atraumatic simple extraction. This study recommends that the use of nanobone in extractions operations and bone fractures lead to speed healing

\section{INTRODUCTION}

-Inorganic biomaterials based on calcium orthophosphate have their wide range of applications in medicine [1-4].

-Hydroxyapatite (HAp), chemical formula Ca5 (PO4)3OH, is considered the structural template for the mineral phase of bone, dentin and enamel. Synthetic HAp is widely used in medicine and dentistry because of its biocom- patibility and bioactivity properties. [5].

Hydroxyapatite has been used to fill a wide range of bony defects in orthopedic and maxillofacial surgeries and dentistry [6-9].

-Hydroxy apatite is the main inorganic components of living hard tissues. Due to its excellent biocompatability, it is regarded as an important bioceramic material in artificial replacement of bones and teeth [10].

Tooth extraction inevitably has influence in bone resorption and changes in gingival contours. Furthermore treatment may become more complex in using dental implants and common prosthetics. Marginal alveolar bone ridge protection has influence in achieving optimal functional, aesthetic prosthesis and orthodontic treatment results. There is increasing demand in lowering damage to soft 


\section{W.Ghanem et al.}

and hard tissues around the tooth being extracted. A traumatic tooth extraction and further protection of alveolus is important [11].

Maintenance of viable alveolar bone and ridges is thought to be dependent on stimulation by the periodontal membrane and the presence of teeth or roots. The healing of the extraction socket in humans occurs in approximately two months, but bone may still grow in a coronal direction up to four or five months, while further maturation of the newly formed bone is completed between six and twelve months after the extraction[12].

This big challenge for the oral surgeon to restore the bone loss and function in the area of missing tooth. So the researches focused on the materials that can be used to restore the bone loss and functions. There are many different types of materials can be used to restore bone defect as autogenous bone graft, allograft, xenograft and alloplast materials.

The recent researches establish the new materials that reduce the crestal bone resorption, accelerate healing process and reduce the vertical and horizontal bone resorption in the area of missing tooth after extraction, so enable the operator freely to make fixed or removable appliance to restore the function and esthetic of the missing tooth area.

In our present study used the Nano-hydroxyapatite after tooth extraction to evaluate hard tissue healing following application of bone substitutes (Nano bone) into fresh extraction sockets in dogs.

\section{MATERIALS AND METHODS}

The study was conducted on twenty eight mature (ranged from 1-2 years old) male Mongrel dogs, weighing about $15-20 \mathrm{~kg}$ each.

The dogs were clinically examined to rule out the presence of any disease and kept under clinical observation for three weeks preoperatively and all dogs fed cooked meat, bread, milk and water. The animals were housed in separate cages, supplied with food and a bucket for water and allowed to live in optimal conditions. All animals subjected to the surgical procedures at the Department of Veterinary surgery, Anesthesiology and Radiology, Faculty of Veterinary Medicine, Suez Canal University.

The animals were divided into three groups:

Group I (control group): consisted of four animals with extracted lower $2^{\text {nd }}$ premolar.

Group II: consisted of twelve animals; the animals in this group were treated with nano hydroxyapatite (NanoBone $\left.{ }^{\circledR}\right)$ to seal the socket after simple atraumatic extraction of lower $2^{\text {nd }}$ premolar.

Group III: consisted of twelve animals; the animals in this group were treated with nano hydroxyapatite (NanoBone ${ }^{\circledR}$ ) with platelet rich fibrin(PRF) to seal the socket after simple a traumatic extraction of lower $2^{\text {nd }}$ premolar.

\subsection{Preoperative Preparation}

The cages were sprayed with 6/1000 Neocidal* (Diazinone) Also the animals were injected by Ivermectine $*$ (Ivomec) $0.1 \mathrm{mg} / \mathrm{kg}$ body weight subcutaneously to guard against ectoparasiytic and endoparasitic infestations. Twelve hours before the operation, Food and water were kept away from the animals to prepare them for anesthesia. Intravenous Injection of prophylactic antibiotic $-1 \mathrm{gm}$ amoxicillin (Flumox 1gm) - administrated to each dog for one day before surgery.

\subsection{Surgical Protocol}

\subsubsection{Anesthesia procedures}

The operation performed under general anesthesia according to the following protocol:

A. All animals were premedicated preoperatively by subcutaneous administration of Atropine sulphate $0.005 \mathrm{mg} / \mathrm{kg}$ body weight $10-30$ minutes prior to surgery.

B. Cannulation of the cephalic vein using 20 gauges: IV cannula

C. Dogs were administered Neurazine *** ampoule $25 \mathrm{mg} / \mathrm{kg}$ (Chlopromazine HCL) intramuscularly and soft diet were provided for 10 days post-surgery.

\footnotetext{
* Manufacture by El Nasr Co. for Pharmaceuticals, Giza, Egypt.

* Manufacture by Merck, Sharp and Dome, USA.

*** Manufacture by Misr Co. Egypt.
} 
Induction of anesthesia by IV administration of sodium thiopental $2.5 \%$ solution $20-30 \mathrm{mg} / \mathrm{kg}$ via a 20 gauge intravenous cannula through the cephalic vein, one third of administrated dose was injected within 15 second, the reminder was given slowly till loss of pedal and corneal reflexes, constriction of the pupils and development of shallow regular respiration

\subsection{Operative Procedures}

The operative site was scrubbed with Betadine* (Providine Iodine $7.5 \%$ ) and the operation site was isolated with cotton rolls. Then infiltration injection technique is applied in the mucobuccal fold in front of the tooth to be extracted with $(1 \mathrm{ml})$ Mepecaine $^{*}$ (2\% Mepivacaine with 1/20000 Levnordefrin) local anesthesia with vasoconstrictor to decrease bleeding in the site of extracted tooth (lower $2^{\text {nd }}$ premolar). Simple atraumatic extraction of lower $2^{\text {nd }}$ premolar was performed for each $\operatorname{dog}$ with preservation of the soft tissue around the socket to prevent delayed healing. After extraction the socket was inspected to be sure that it was empty of any debris or bony fragments inside it.

Group II nano hydroxyapatite (NanoBone $\left.{ }^{\circledR}\right)^{*}$ mixed with blood gathered from the socket and put the mix inside it until completely filled the extracted socket cavity.

Group III the socket was completely filled by homogeneous mass of (nano hydroxyapatite (NanoBone ${ }^{\circledR}$ ) and platelet rich fibrin $\{\mathrm{PRF}\}$ ).

\subsection{Preparation of Platelet Rich Fibrin (PRF)}

$10 \mathrm{ml}$ venous blood in tubes (free from anticoagulant) was suddenly centrifuged at $3000 \mathrm{rpm}$ for 10 minutes. In a few minutes, in the sample there is an activation of a great amount of platelets after coagulation reactions born with tube contact. After centrifugation, a cellular plasma supernatant, or PPP (Platelet-Poor-Plasma), will be found at the top of the tube, PRF in the middle and blood cells at the bottom. Mass was pulled by twizzer from tube and PRF separated and mix with (NanoBone®) until became homogenous mass and put carefully inside the socket until completely filled the whole extracted socket.

All extracted bony sockets were completely closed by suturing the overlying soft tissue with absorbable suture material Vicry1000* (polyglactin).

\subsection{Postoperative Care}

For postoperative pain relief, an intramuscular injection of voltaren ${ }^{*} *$ was given during the first 24 hours, and $1 \mathrm{gm}$ amoxicillin (Flumox $1 \mathrm{gm}$ ) ${ }^{* *} *$ intravenously for four days to reduce pain and swelling The animals were checked daily for the first post operative week for signs of infections and were fed with soft consistency diet.

\subsection{Animals Sacrifice}

One animal from group I and three animals from group II and III were sacrificed with an overdose of anesthesia at 15, 30,45and 90 day. The mandible was freed from their attachment tissues. The specimens (area of extracted socket $2^{\text {nd }}$ premolar) were placed in $10 \%$ formalin solution. Following fixation, decalcification of the block sections was accomplished by immersion in $20 \%$ nitric acid approximately 20 days. Dehydration followed by paraffin embedding and microtome serial sectioning $(5 \mu)$ was performed. Slides were stained with Hematoxylin and Eosin and examined by light microscope.

\section{RESUlTS}

All dogs in the present study were check out and the data that were recorded did not reveal missing of any dog through the whole study intervals and simple atraumatic extraction of one tooth did not affect on the dog desire for eat. Clinically, all of the 28 extraction sockets healed uneventfully. Soft tissue healing preceded normally no signs of inflammation or infection. By the end of the 12 weeks of the study all sites were clinically healed completely.

\footnotetext{
* Manufacture by Nile Co. for Pharmaceuticals, Cairo.

* Manufacture by Alexandria pharmaceuticals. Alexandria, Egypt.

* Artoss GmbH, 18119 Rostock, Germany.

* Johnson \& Johnson/ Ethicon, Somerville, $\mathrm{Nj}$.

** Diclofenac sodium, manufactured by, Novartis pharma.S.A.E Cairo, Egypt.

*** Manufacture by Eipico Co.
} 


\subsection{Microscopic Examination}

\subsubsection{After 15 days}

\section{Group I (Control Group)}

Histological sections stained with (H\&E) showed socket containing small amount of granulation tissue that was composed of newly formed capillaries \& fibrous connective tissue in addition to few numbers of inflammatory cells mainly macrophages and lymphocytes. (Fig. 1)
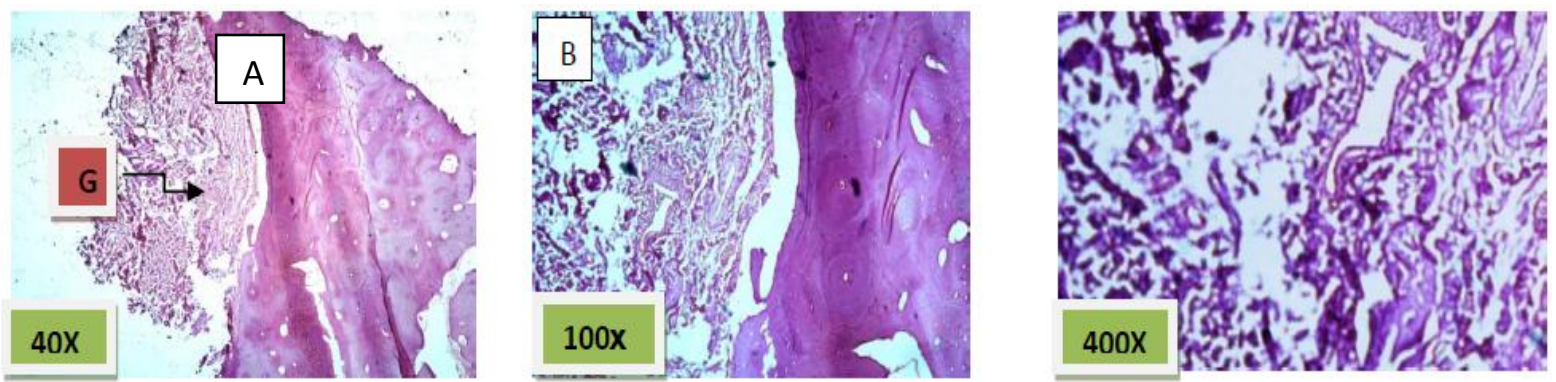

Fig1. Control group (group I): A) the socket is partially filled with granulation tissue $(\boldsymbol{G}), H \& E 40 X$.

$\boldsymbol{B}) \boldsymbol{\&} \boldsymbol{C})$ higher magnification of the previous image showing fibrous connective and the newly formed capillaries (white arrow) $H \& E 100 x$ and 400x, respectively.

Group II ( Nanobone alone)

Histological sections stained with (H\&E) showed socket completely filled with granulation tissue. (Fig. 2)
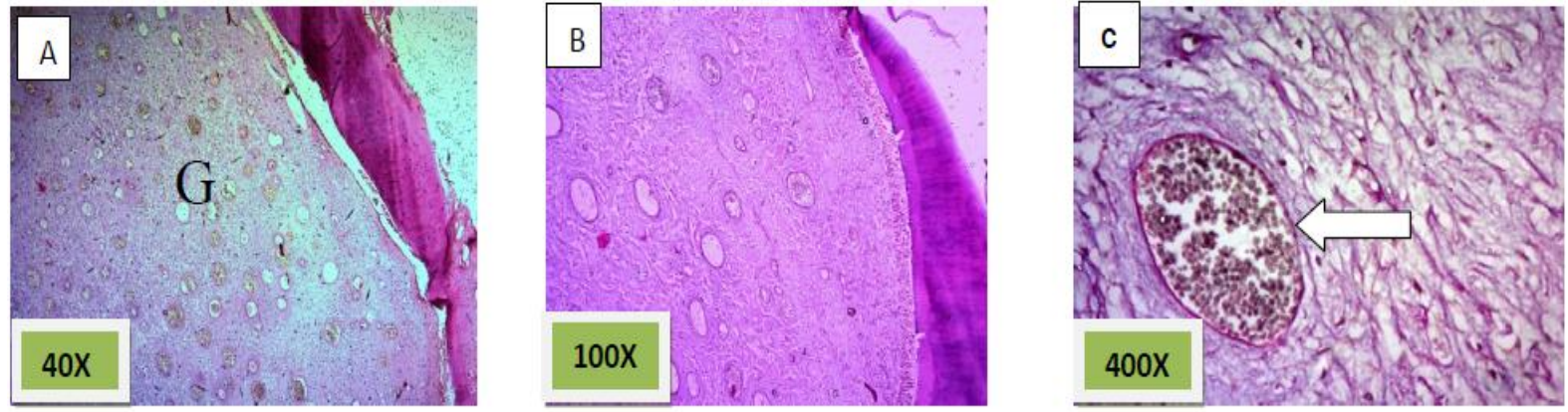

Fig2. Group II (Nanobone alone): $\boldsymbol{A})$ the socket is completely filled with granulation tissue $(G), H \& E 40 X$. B) $\boldsymbol{\&} \boldsymbol{C})$ higher magnification of the previous image showing fibrous connective and the newly formed capil.. ries (white arrow) H\&E 100x and 400x, respectively

Group III ( Nanobone with PRF)

Histological sections stained with (H\&E) showed the formation of newly formed bone (woven bone) at the periphery of the socket which projecting from inter-septal bone toward the center of the socket intermixed with fibrous connective tissue. The woven bone was lined the lining plump osteoblasts .There was low number of inflammatory cells (lymphocytes\& macrophage). (Fig. 3)
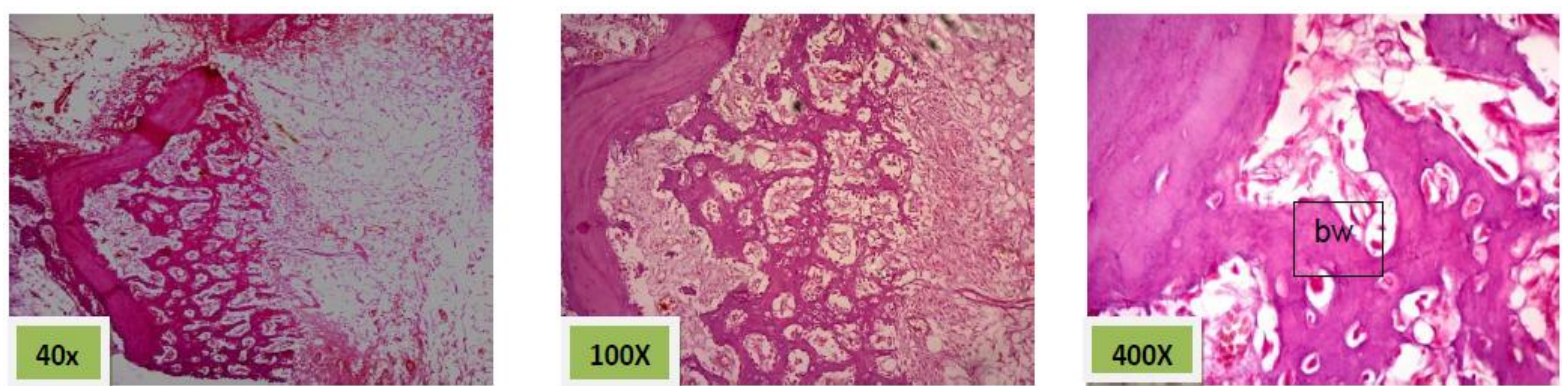

Fig3. Group III (Nanobone +PRF): $\boldsymbol{A})$ the socket is filled with granulation tissue $(\boldsymbol{G})$ in addition to newly formed bone, $H \& E 40 X . B) \& C$ ) higher magnification of the previous image showing the woven bone(WB) projecting from the inter-septal bone (S) H\&E 100x and 400x, respectively. 


\subsubsection{After 30 days}

\section{Group I (Control Group)}

Histological sections stained with (H\&E) showed socket containing mature fibrous connective tissue. (Fig. 4)
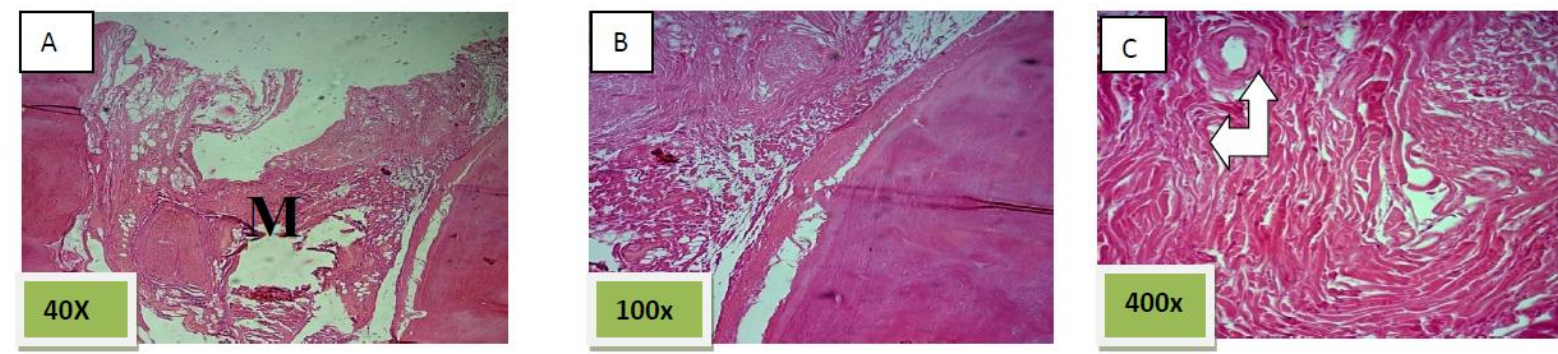

Fig4. Control group(group I): A) the socket is filled with mature fibrous connective tissue(M),H\&E 40X. B) $\boldsymbol{\&} \boldsymbol{C})$ higher magnification of the previous image showing fibrous connective tissue and the newly formed capillaries (white arrow) H\&E 100x and 400x, respectively.

\section{Group II ( Nanobone alone)}

Histological sections stained with (H\&E) showed $50 \%$ of the socket was filled with woven bone (slightly basophilic bone with many osteocyte haphazardly arranged).the rest of socket was connective tissue mixed with inflammatory cells (lymphocyte\&macrophage). (Fig. 5)
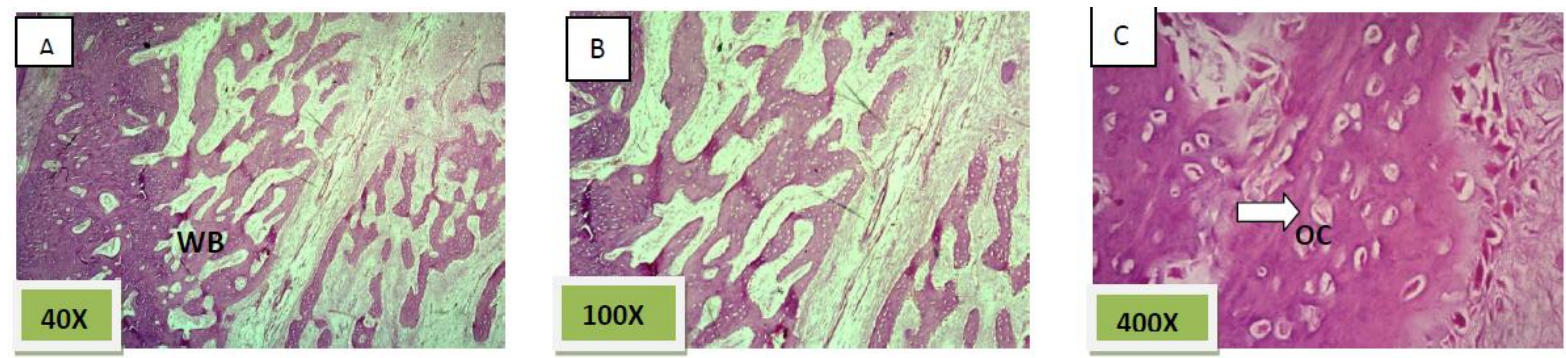

Fig5. Group II (Nanobone alone): $\boldsymbol{A})$ the socket is filled with spicules of woven bone (WB) and connective tissue,$H \& E 40 X . B) \& C$ ) higher magnification of the previous image showing slightly basophilic bone with many osteocytes $(\mathrm{OC})$ (white arrow) haphazardly arranged. The rest of tissue was connective tissue mixed with inflammatory cells (lymphocyte\& macrophage) H\&E 100x and 400x, respectively.

Group III ( Nanobone with PRF )

Histological sections stained with (H\&E) showed the majority of the socket was filled with woven bone nearly $90 \%$ with few amounts of fibrous connective tissue (Fig. 6).
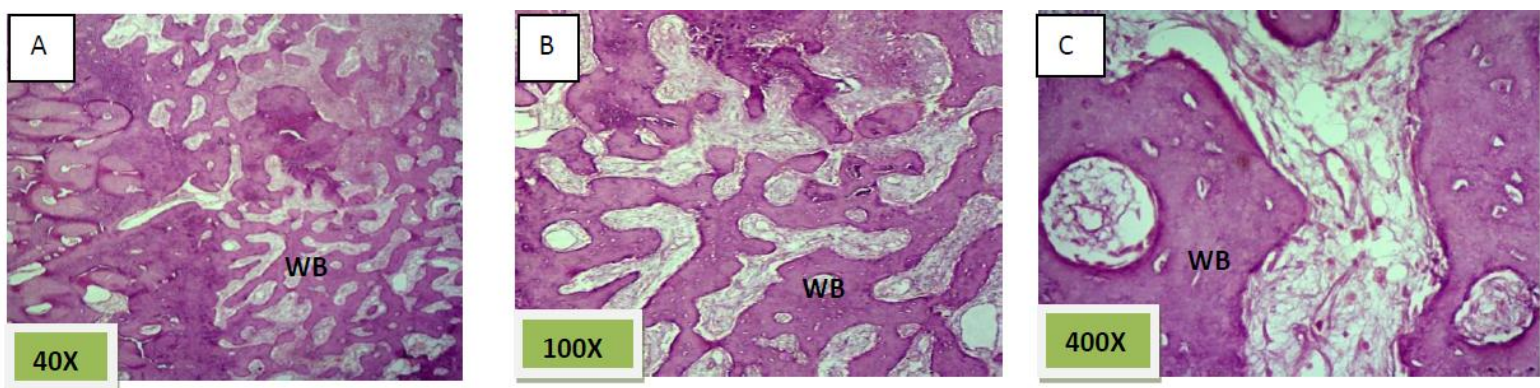

Fig6. Group III (Nanobone +PRF): A) the socket is filled with woven bone (WB) nearly $90 \%$ with few amounts of fibrous connective tissue. , $H \& E 40 X . B) \& C$ ) higher magnification of the previous image showing the spicules of woven bone $H \& E 100 x$ and 400x, respectively.

\subsubsection{After 45 days}

\section{Group I (Control Group)}

Histological sections stained with (H\&E) showed socket filled with spicules of woven bone $(50 \%)$ mixed with mature fibrous connective tissue. (Fig. 7) 

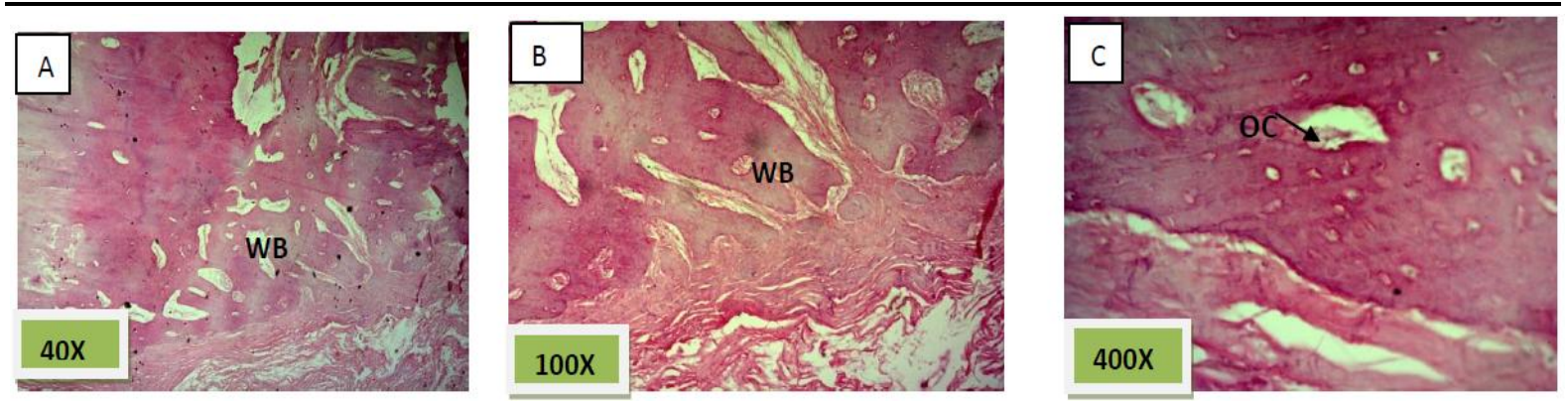

Fig7. Control group (group I): A) the socket is filled with spicules of woven bone(WB) (50\%) mixed with mature fibrous connective tissue., $H \& E 40 X . \boldsymbol{B}) \& C$ ) higher magnification of the previous image showing fibrous connective tissue between the bony spicules which contained osteocyte $(\mathrm{OC})$ haphazardly arranged $H \& E 100 x$ and $400 x$, respectively.

\section{Group II ( Nanobone alone)}

Histological sections stained with (H\&E) showed socket is filled with woven bone with more wider spicules that are more ordinary fused to each other except of few area occupied by fibrous connective tissue, (Fig. 8)
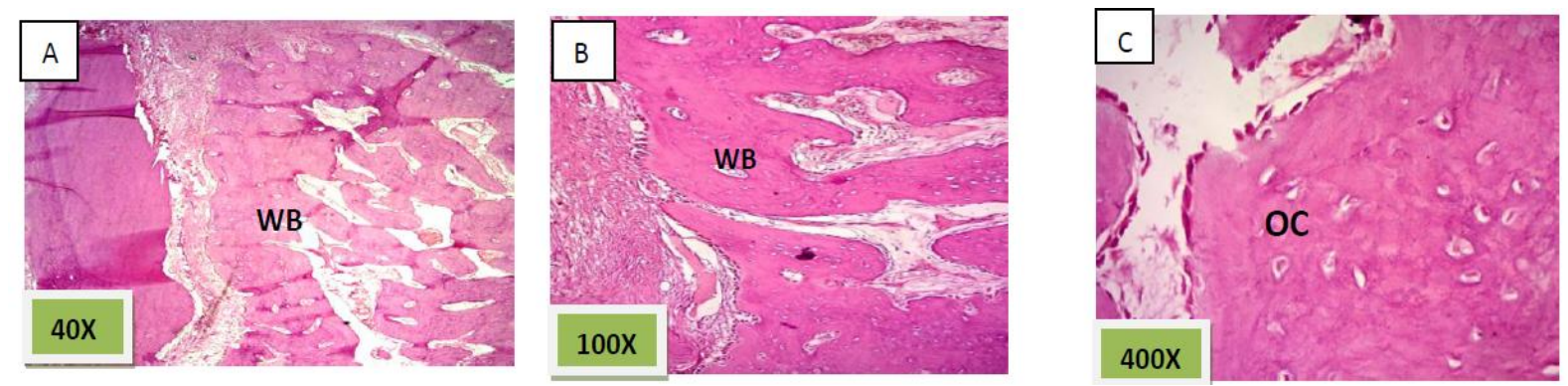

Fig.8. Group II (Nanobone alone): A) nearly all the socket is filled with spicules of woven bone (WB) and mature fibrous connective tissue founded in few areas, $H \& E 40 X . B) \& C$ ) higher magnification of the previous image showing woven bone (WB) with more wider spicules that are more ordinary fused to each other with many osteocytes (OC) H\&E 100x and 400x, respectively.

\section{Group III (Nanobone with PRF)}

Histological sections stained with (H\&E) showed nearly all the socket is filled with bony spicules that are contain moderate number of osteocyte and few amount of bone marrow within the space. (Fig. 9)
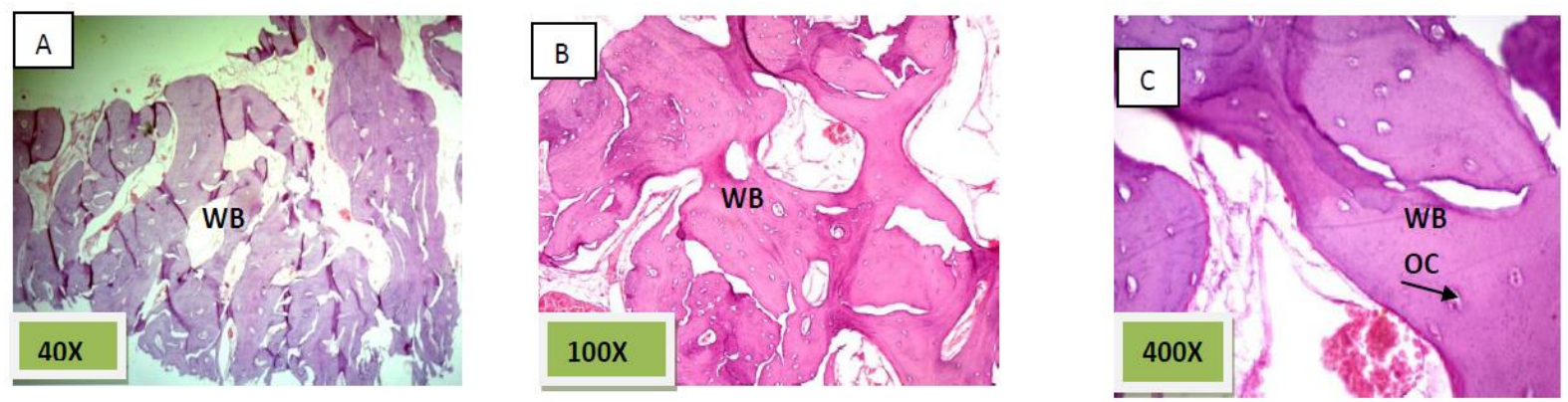

Fig9. Group III (Nanobone +PRF): $\boldsymbol{A})$ nearly all the socket is filled with wide woven bony spicules(WB) with few amounts of fibrous connective tissue. , $H \& E 40 X . B) \& C$ ) higher magnification of the previous image showing bony spicules that are contain moderate number of osteocyte (OC) and few amount of bone marrow within the space. $H \& E 100 x$ and 400x, respectively.

\subsubsection{After 90 days}

\section{Group I (Control Group)}

Histological sections stained with (H\&E) showed socket filled with lamellar bone with wide marrow spaces. (Fig. 10) 

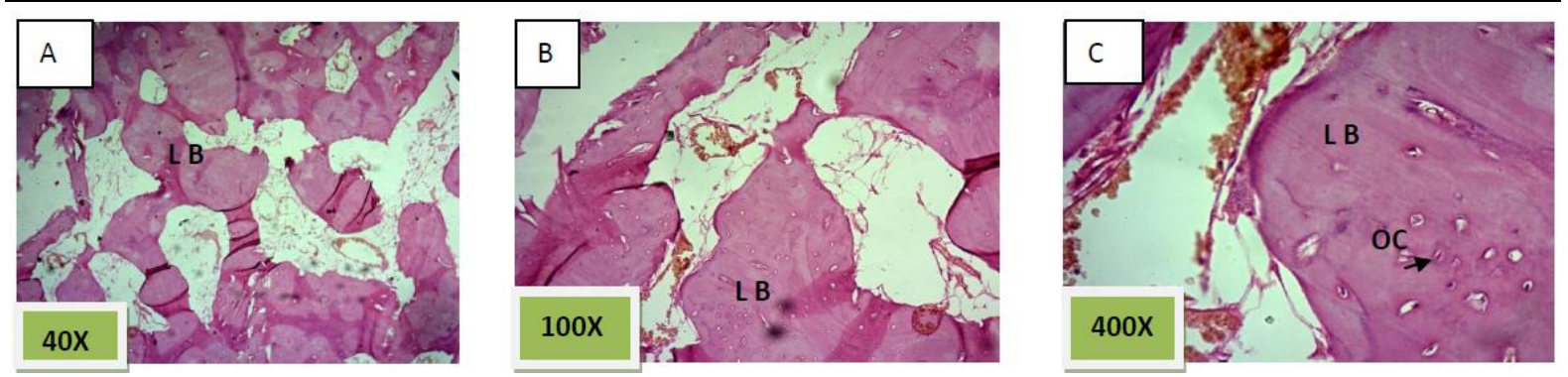

Fig10. Group (group I): A) the socket is filled with lamellar bone(L B) with wide marrow spaces ., $H \& E 40 X$. $\boldsymbol{B}) \& \boldsymbol{C})$ higher magnification of the previous image showing osteocyte $(\boldsymbol{O C})$ and marrow spaces and its contents $H \& E 100 x$ and $400 x$, respectively.

\section{Group II ( Nanobone alone)}

Histological sections stained with (H\&E) showed the socket filled with lamellar bone except of few areas occupied with marrow spaces (Fig.11).
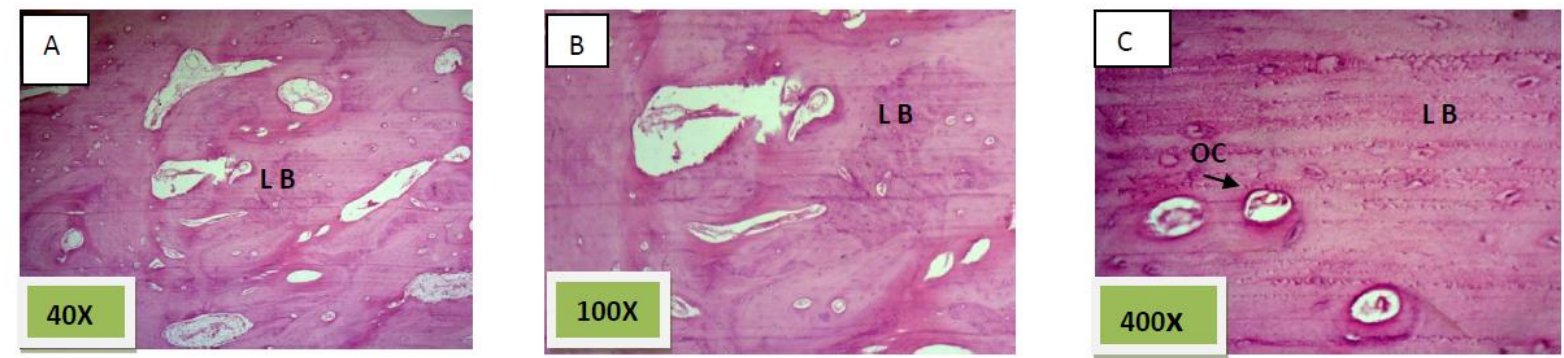

Fig11.Group II (Nanobone alone): $\boldsymbol{A})$ all the socket is filled with lamellar bone (LB) and marrow spaces founded in few areas, $H \& E 40 X . B) \& C$ ) higher magnification of the previous image showing lacunae of osteocyte and haversian system (OC) $H \& E$ 100x and 400x, respectively.

Group III (Nano bone with PRF)

Histological sections stained with (H\&E) showed socket filled with thick well defined lamellar bone. (Fig. 12)
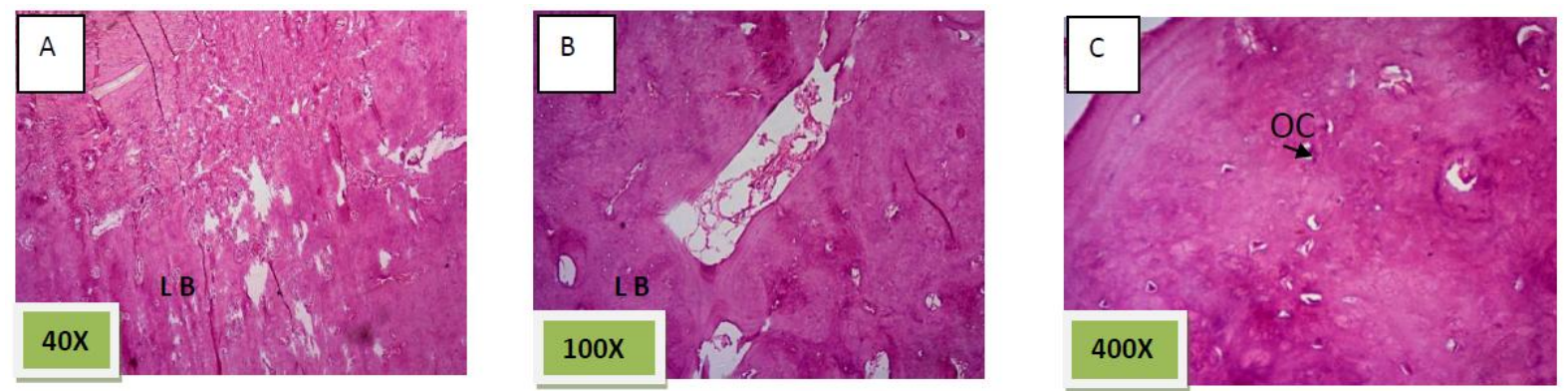

Fig12. Group III (Nanobone +PRF): $\boldsymbol{A})$ all the socket is filled with thick and well-defined lamellar bone (LB) , $H \& E 40 X . B) \& C)$ higher magnification of the previous image showing lacunae of osteocyte(OC) and haversian system $H \& E 100 x$ and $400 x$, respectively.

\section{DisCuSSION}

After tooth extraction, a significant reduction of the alveolar ridge in the horizontal/buccal-palatal dimension occurs if the socket does not receive some type of treatment; the bone filling of the socket can be significantly improved with preservation techniques. The maturation and mineralization of the newly formed bone in the extraction socket can be accelerated or improved by graft materials[13]. (Mezzomo et al 2011)

NanoBone ${ }^{\circledR}$ is a recently developed and approved granular material consisting of nanocrystalline HA embedded in a silica gel matrix [14]. (Gerike et al 2006)

This offers several advantages of nanostructural biomaterials [15]. (Webster and Ahn 2006)

Park (2009) ; Wikesjö et al (2009)[16,17] suggested that combining growth factors (PRF) with osteoconductive scaffolds may facilitate a faster and more significant enhancement of new bone formation of high quality thanks to the delivery of the growth factors at the site of the graft. 


\section{W.Ghanem et al.}

Choukroun et al (2006)[18] reported encouraging results, by using growth factor- platelet riched fibrin(PRF) for bone regeneration in sinus lift. The histological analysis showed a bone growth and a better trabecular organization in areas treated with PRF and freeze dried bone allograft (FDBA), compared to control areas which where only treated with FDBA. The rate of vital bone/inert bone of the trabecular bone, allows to appreciate the importance of a turnover.

These results are agreed with the histological results of group III (Nanobone with PRF) which were showed the greatest rate of newly bone formation compared to group II (Nanobone alone) and group I (Control group).While the quality of newly formed bone was similar for group II (Nanobone alone) and group III (Nanobone with PRF) but greater than that of group I (Control group).

\section{CONCLuSiON}

\section{From the current study we concluded the followings}

i Nanobone graft is a compatible material and doesn't interfere with the healing process of extracted bony socket.

ii Nanobone graft material support and accelerate the formation of bone filling the extracted bony socket.

iii Nanobone graft provides the good quality of newly formed bone that fill the extracted bony socket.

iv Nanobone grafts with the growth factor Platelet Rich Fibrin (PRF) accelerate the rate of bone formation that fill the extracted bony socket more than Nanobone graft alone or without.

v Nanobone graft alone and Nanobone graft with the growth factor Platelet Rich Fibrin (PRF) preserved three dimensions of extracted bony socket after atraumatic simple extraction.

\section{REFERENCES}

[1] Dorozhkin SV: Calcium orthophosphates in nature, biology and medicine. Materials 2009, 2:399-498.

[2] Vallet-Regí M, González-Calbet JM: Calcium phosphates as substitution of bone tissues. Progress Solid State Chem 2004, 32:1-31.

[3] Dorozhkin SV: Nanodimensional and nanocrystalline apatites and other calcium orthophosphates in biomedical engineering, biology and medicine. Materials 2009, 2:1975-2045.

[4] Chen F, Zhu Y-J, Zhang K-H, Wu J, Wang K-W, Tang Q-L, Mo X-M: Europium-doped amorphous calcium phosphate porous nanospheres: preparation and application as luminescent drug carrier. Nanoscale RLett 2011, 6:6.es

[5] (Zapanta LeGeros, R. (1991) Calcium phosphates in oral biology and medicine. Karger, New York.).

[6] Hench LL: Bioceramics. J Am Ceram Soc 1998, 81:1705-1728.

[7] Friedman CD, Costantino PD, Snyderman CH, Chow LC, Takagi S: Reconstruction of the frontal sinus and frontofacial skeleton with hydroxyapatite cement. Arch Facial Plast Surg 2000, 2:124-129.

[8] Zakharov NA, Polunina IA, Polunin KE, Rakitina NM, Kochetkova EI, Sokolova NP, Kalinnikov VT: Calcium hydroxyapatite for medical applications. Inorg Mater 2004, 40:641648.

[9] Verret DJ, Ducic Y, Oxford L, Smith J: Hydroxyapatite cement in craniofacial reconstruction. Otolaryngol Head Neck Surg 2005, 133:897-899.

[10] Fanovish, M.A., et al. (1998) Improvement of micro struc- ture and micro hardness of hydroxyapatite ceramics by addition of lithium. Material Letters, 33, 269-272.

[11] Marius K., Ricardas K., Alvydas G.: The preservation of alveolar bone ridge during tooth extraction. Stomatol, Balt Dent and Maxillofac J, 2012; 14: 3-11

[12] Evian CI, Rosenberg ES, Coslet JG, Corn H.: The osteogenic activity of bone removed from healing extraction sockets in humans. J Periodontol, 1982; 53(2): 81- 85.

[13] Mezzomo LA., Shinkai RS., Mardas N., Donos N.: Alveolar ridge preservation after dental extraction and before implant placement. Rev Odonto Cienc 2011;26(1):77-83 
[14] Gerike W.,Bienengra“ ber V., Henkel KO., Bayerlein T., Proff P., Gedrange T., Gerber T.: The manufacture of synthetic non-sintered and degradable bone grafting substitutes. Folia Morphol (Warsz). 2006;65: 54-55

[15] Webster TJ. and Ahn ES.: Nanostructured biomaterials for tissue engineering bone. Advances in Biochem Eng Biotechnol,2006;103: 275-308

[16] Park JB.: Use of bone morphogenetic proteins in sinus augmentation procedure. $\mathrm{J}$ of Cranio facial Surg, 2009; 20(5):1501-3.

[17] Wikesjö UM., Qahash M., Huang YH., Xiropaidis A., Polimeni G., Susin C.: Bone morphogenetic proteins for periodontal and alveolar indications; biological observationsclinical implications. Ortho Craniofaci Res, 2009;12(3):263-70

[18] Choukrou N., Diss A., Simonpieri A., Girard MO., Schoeffler C., Dhoan SL., Dhoan AJ., Mouhyi J.,Dhoan DM.: Platelet-rich fibrin (PRF): a second generation platelet concentrate. Part IV: clinical effects on tissue healing. Oral Surg, Oral, MedOral, Pathol Oral, Radiol Endod, 2006; 101: e56-60. 